\title{
Contents
}

\section{Introduction to Neutron Techniques}

Götz Eckold and Helmut Schober............................ 1

1.1 Why Neutrons? .............................. 1

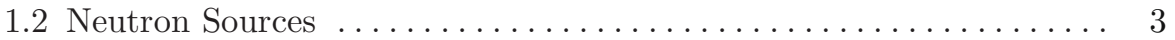

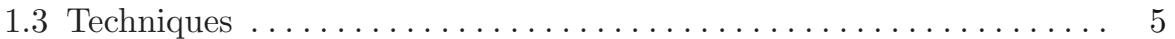

1.3.1 Three Axis Spectrometers..................... 6

1.3.2 Backscattering Spectrometers .................... 7

1.3.3 Time-of-Flight Spectrometers $\ldots \ldots \ldots \ldots \ldots \ldots \ldots \ldots \ldots . . \ldots$

1.3.4 Fixed Wavelength Diffractometers .................. 11

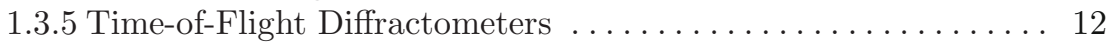

1.3.6 SANS Instruments . . . . . . . . . . . . . . . . . . . 12

1.3.7 Reflectometers ............................ 14

1.3.8 Spin-Echo Spectrometers . . . . . . . . . . . . . . . . . . 15

$1.4 \ldots$ and What About Kinetics? . . . . . . . . . . . . . . . . . . . 17

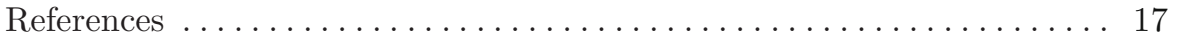

2 Studying the Hydration of Cement Systems in Real-time Using Quasielastic and Inelastic Neutron Scattering

Vanessa K. Peterson ...................................... 19

2.1 Cement Research ................................... 19

2.1.1 Constituents and Hydration ...................... 20

2.1 .2 Hydration Kinetics . . . . . . . . . . . . . . . . . . . . 21

2.1 .3 Research Tools ............................. 22

2.2 Studying Hydrating Cement Using Quasielastic and Inelastic Neutron Scattering $\ldots \ldots \ldots \ldots \ldots \ldots \ldots \ldots . \ldots . \ldots . \ldots . \ldots$

2.2.1 Quasielastic Neutron Scattering of Hydrogen

in Cement Systems ............................ 26

2.2.2 Models for QENS Data ....................... 26

2.2.3 Inelastic Neutron Scattering of Hydrogen

in Cement Systems ............................ 40

2.2.4 Summary of QENS and INS methods $\ldots \ldots \ldots \ldots \ldots \ldots . .46$ 
2.3 Time-Resolved Quasielastic and Inelastic Neutron Scattering . . . . . . 47

2.3.1 Time-evolution of Descriptive Parameters Derived

from Quasielastic and Inelastic Neutron Scattering Data . . . . . 47

2.3.2 Kinetic Models . . . . . . . . . . . . . . . . . . . . . . 57

2.3.3 The Kinetics of Cementitious Hydration using Quasi

and Inelastic Neutron Scattering: Case Studies . . . . . . . . . 65

2.4 Conclusions and Outlook ...................... 73

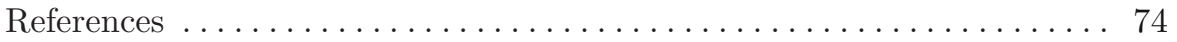

3 Kinetic Properties of Transformations Between Different

Amorphous Ice Structures

Michael Marek Koza, Thomas Hansen, Roland P. May,

Helmut Schober................................ 77

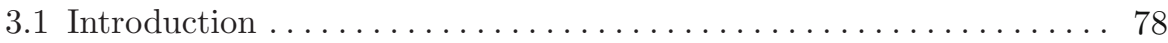

3.2 Experimental . . . . . . . . . . . . . . . . . . 81

3.2.1 Sample Preparation and Experimental Procedure ........ . 81

3.2 .2 Data Treatment .......................... 83

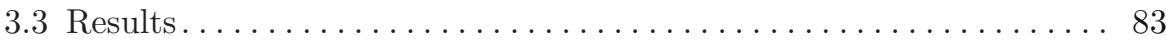

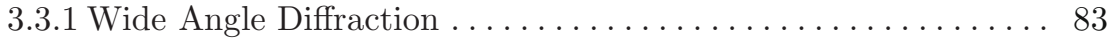

3.3 .2 Small Angle Signal . . . . . . . . . . . . . . . . . . . . . . . . . . 86

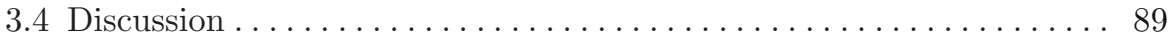

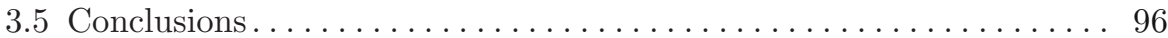

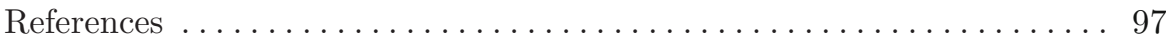

4 Structure Evolution in Materials Studied

by Time-Dependent Neutron Scattering

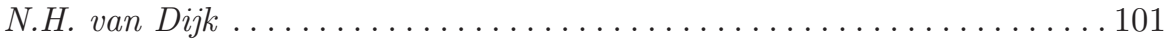

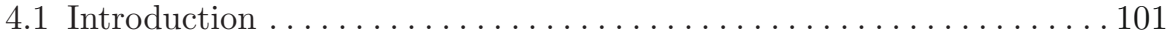

4.2 Kinetics of Phase Transformations . . . . . . . . . . . . . . . . . 102

4.3 Time-Resolved Neutron Scattering Techniques . . . . . . . . . . . . . . 103

4.3.1 Characteristics Neutron Scattering Techniques

and Measurement Strategies .................... 103

4.3.2 Comparison Neutron and Synchrotron Studies . . . . . . . . . . . 105

4.4 Neutron and X-ray Studies During Solidification

of Aluminium Alloys . . . . . . . . . . . . . . . . . . . . . 106

4.4.1 Time Resolved Neutron Scattering Experiments . . . . . . . . . . . 106

4.4.2 Time Resolved X-ray Scattering Experiments . . . . . . . . . . . . . 109

4.5 3D Neutron Depolarization Studies . . . . . . . . . . . . . . . . . . . 111

4.5.1 Time-Resolved Magnetic Domain Wall Movement... . . . . . . . . 112

4.5.2 Time-Resolved Phase Transformation Kinetics in Steels . . . . . . . 114

4.6 Spin-Echo Small-Angle Neutron Scattering . . . . . . . . . . . . . . 117

4.7 Conclusions and Prospects . . . . . . . . . . . . . . . . . . . . . . 120

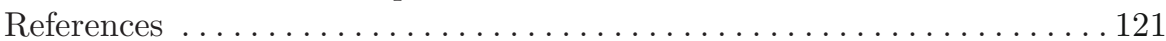




\section{Applications of In Situ Neutron Diffraction}

to Optimisation of Novel Materials Synthesis

D.P. Riley, E.H. Kisi, E. Wu, T. Hansen, P. Henry............... 123

5.1 Brief Review of In Situ Diffraction

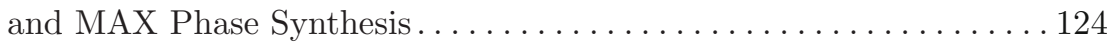

5.1 .1 Introduction to In Situ Diffraction . . . . . . . . . . . . . . 124

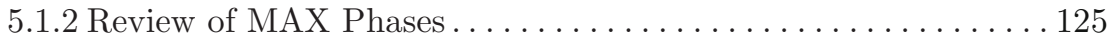

5.2 In situ Neutron Diffraction: Long Time Scales . . . . . . . . . . . . . . 127

$5.2 .1 \mathrm{Ti}_{3} \mathrm{SiC}_{2}$ Reactive Sintering Synthesis Mechanism . . . . . . . . . 127

$5.2 .2 \mathrm{Ti}_{3} \mathrm{AlC}_{2}$ Reactive Sintering Synthesis Mechanism . . . . . . . . . . 129

$5.2 .3 \mathrm{Ti}_{3} \mathrm{SiC}_{2}$ Synthesis Kinetics . . . . . . . . . . . . . . . 130

5.3 In situ Neutron Diffraction: Short Time Scales . . . . . . . . . . . . . . 132

$5.3 .1 \mathrm{Ti}_{3} \mathrm{SiC}_{2}$ SHS Synthesis Mechanism . . . . . . . . . . . . . 132

5.3.2 In situ Diffraction Differential Thermal Analysis . . . . . . . . . . 134

5.4 Designer Processing Routes from In Situ Neutron Diffraction

Analysis . . . . . . . . . . . . . . . . . . . . . . . . . . . . 135

5.4 .1 Inter-Conversion of MAX Phases . . . . . . . . . . . . 135

5.4.2 Intercalation of the A Element into a Crystalline Precursor . . 136

5.4 .3 Lessons Learned . . . . . . . . . . . . . . . . . . . . . . . . 139

5.5 Design of Future In Situ Diffraction Equipment . . . . . . . . . . . . . 140

5.5.1 In Situ Diffraction Chamber Design (Institutional) . . . . . . . . 141

5.5.2 In Situ Reaction Chamber Design (User Inserts) . . . . . . . . . . . 144

5.5.3 Assembled ISRC Design . . . . . . . . . . . . . . . . . . . 145

References ................................. 147

\section{Time-Resolved, Electric-Field-Induced Domain Switching and Strain in Ferroelectric Ceramics and Crystals}

Jacob L. Jones, Juan C. Nino, Abhijit Pramanick,

and John E. Daniels ................................... 149

6.1 Introduction . . . . . . . . . . . . . . . . . . . . . . . . 149

6.1.1 Piezoelectricity, Ferroelectricity, and Device Applications . . . . 149

6.1.2 Time-Resolved Neutron Scattering . . . . . . . . . . . . . . . . . . 152

6.1.3 Stroboscopic Techniques . . . . . . . . . . . . . . . . . 153

6.2 Experimental . . . . . . . . . . . . . . . . . . . . . 154

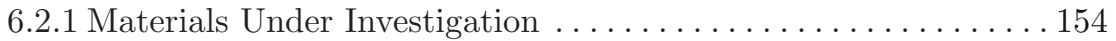

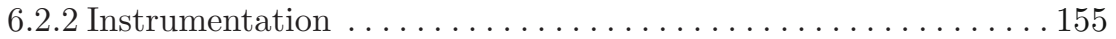

6.3 Domain Wall Motion in Ferroelectric Ceramics . . . . . . . . . . . . . . 157

6.3.1 Application of Static Electric Fields . . . . . . . . . . . . . . 157

6.3.2 Application of Subcoercive, Periodic Electric Fields . . . . . . . . 159

6.4 Time-Resolved Studies of Lattice Strain in Ferroelectric Ceramics . . 161

6.5 Domain Switching and Strain in Ferroelectric Relaxor

Single Crystals . . . . . . . . . . . . . . . . . . . . . . . . . . 164 
6.6 Future Opportunities and Outlook for Time-Resolved Scattering

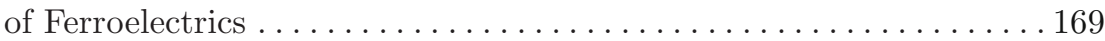

6.6.1 Instrumentation Developments . . . . . . . . . . . . . . . . 170

6.6.2 Application to other Structures and Phenomena . . . . . . . . . 171

6.6.3 Correlation Between Macroscopic Properties

and Diffraction Measurements . . . . . . . . . . . . . . . . . . 172

References .................................... 173

\section{Time-Resolved Phonons as a Microscopic Probe for Solid State Processes}

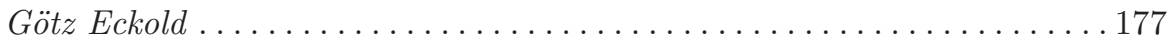

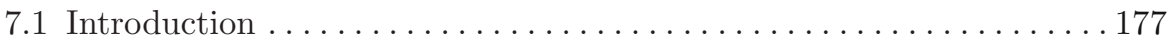

7.2 Techniques . . . . . . . . . . . . . . . . . . . . 178

7.3 Kinetics Between Seconds and Years: Demixing Processes in

Simple Systems . . . . . . . . . . . . . . . . . . . . . . 181

7.3.1 Basics of Demixing and Phase Diagrams

of Silver-Alkali Halides . . . . . . . . . . . . . . . . . . . . . . . 181

7.3 .2 Experimental. . . . . . . . . . . . . . . . . . . . . . . . . . 184

7.3.3 Nucleation and Growth in $\mathrm{KCl}-\mathrm{NaCl}$ Mixed Crystals . . . . . . . . 184

7.3.4 Spinodal Decomposition in $\mathrm{AgCl}-\mathrm{NaCl}$ Mixed Crystals . . . . . . . 184

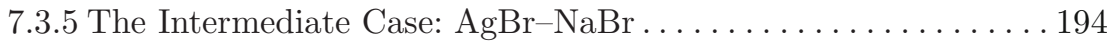

7.4 Kinetics in the Microsecond Regime: Phase Transitions in

Ferroelectrics . . . . . . . . . . . . . . . . . . . . . 203

7.4.1 Modulated Ferroelectrics and Softmode Transitions . . . . . . . . 203

7.4 .2 Experimental . . . . . . . . . . . . . . . . . . . . . . . . . . 204

7.4.3 The Lock-in Transition in $\mathrm{K}_{2} \mathrm{SeO}_{4} \ldots \ldots \ldots \ldots \ldots \ldots$

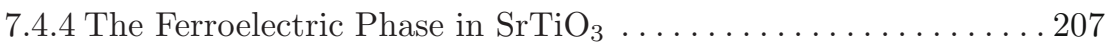

7.5 Concluding Remarks and Future Prospects for Time-Resolved

Inelastic Scattering . . . . . . . . . . . . . . . . . . . . . . 208

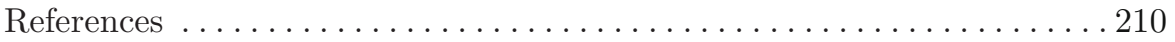

\section{Small Angle Neutron Scattering as a Tool to Study Kinetics of Block Copolymer Micelles}

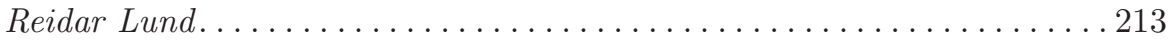

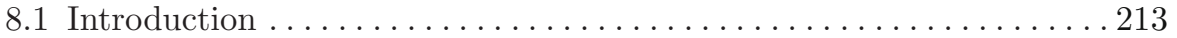

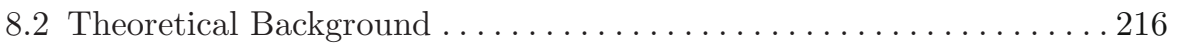

8.2.1 Brief Introduction of Thermodynamics and Scaling Laws . . . . 216

8.2 .2 Aniansson and Wall Mechanism . . . . . . . . . . . . . . 217

8.2.3 Scaling Theory - Halperin and Alexander . . . . . . . . . . 218

8.2 .4 Other Theories . . . . . . . . . . . . . . . . . . . . . . . . 220

8.3 Experimental Background: Small Angle Neutron Scattering . . . . . . . 221

8.3.1 Structure with SANS: Core-Shell Model . . . . . . . . . . . . . 221

8.3.2 Equilibrium Kinetics and Time Resolved SANS . . . . . . . . . . . 224 
8.4 Results - Equilibrium Micellar Kinetics . . . . . . . . . . . . . . 226

8.4.1 Low Molecular Weight Surfactant Micelles . . . . . . . . . . . . 226

8.4 .2 Block Copolymer Micelles . . . . . . . . . . . . . . . . . . . . 227

8.4.3 Amphiphilic Diblock Copolymer Micelles

in Aqueous Solutions . . . . . . . . . . . . . . . . . . 228

8.4.4 Diblock Copolymer Micelles in Organic Solvents . . . . . . . . . . . 233

8.4.5 Triblock Copolymer Micelles in Organic Solvents . . . . . . . . . . 235

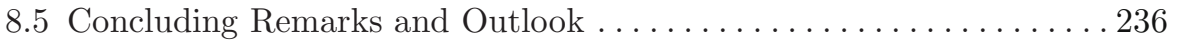

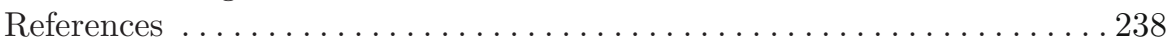

9 Stroboscopic Small Angle Neutron Scattering Investigations of Microsecond Dynamics in Magnetic Nanomaterials

A. Wiedenmann, R. Gähler, R. P. May, U. Keiderling, K. Habicht,

S. Prévost, M. Klokkenburg, B. Erné, and J. Kohlbrecher ........... 241

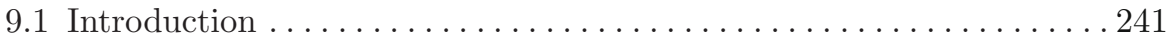

9.2 Stroboscopic SANS Techniques . . . . . . . . . . . . . . . . . . . 242

9.3 Experimental . . . . . . . . . . . . . . . . . . . . . . . . 245

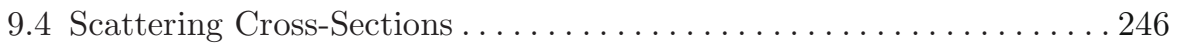

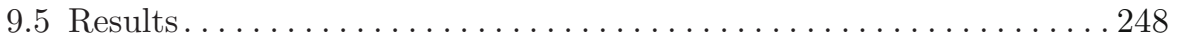

9.5.1 Relaxation of Magnetic Correlations Toward

Equilibrium in Cobalt-FF . . . . . . . . . . . . . . . . 248

9.5.2 Response on Oscillating Field in Continuous

Stroboscopic SANS . . . . . . . . . . . . . . . . . 252

9.5.3 Response from Pulsed Stroboscopic Technique TISANE . . . . . 255

9.5.4 Temperature and Frequency Dependence . . . . . . . . . . . 256

9.5.5 Co-Precipitates in Solid CuCo Alloy . . . . . . . . . . . . . . . . 260

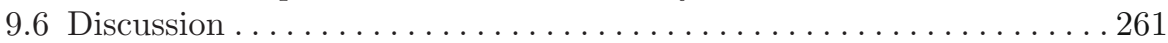

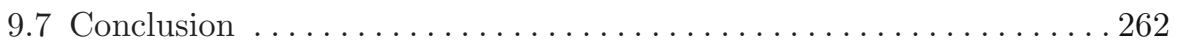

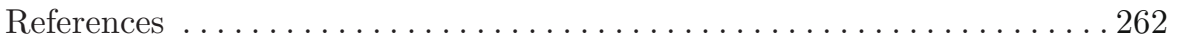

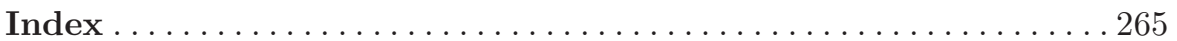

